\title{
Akt/eNOS and MAPK Signaling Pathways Mediated the Phenotypic Switching of Thoracic Aorta Vascular Smooth Muscle Cells in Aging/Hypertensive Rats
}

\author{
Lin ZHANG $^{1 *}$, Zhaoxia XU ${ }^{1 *}$, Ying WU ${ }^{1}$, Jingwen LIAO ${ }^{1}$, Fanxing ZENG ${ }^{1}$, Lijun SHI ${ }^{1}$ \\ * These authors contribute equally to this work. \\ ${ }^{1}$ Department of Exercise Physiology, Beijing Sport University, Beijing, China
}

Received October 16, 2017

Accepted February 15, 2018

On-line May 10, 2018

\begin{abstract}
Summary
Considerable evidence demonstrates that phenotypic switching of vascular smooth muscle cells (VSMCs) is influenced by aging and hypertension. During phenotypic switching, VSMCs undergo a switch to a proliferative and migratory phenotype, with this switch being a common pathology in cardiovascular diseases. The aim of this study was to explore the joint influence of age and hypertension on thoracic aortic smooth muscle phenotypic switching and the balance of Akt and mitogen-activated protein kinase (MAPK) signaling during this switch. Different ages of spontaneously hypertensive rats (SHR) and Wistar-Kyoto rats (WKY) were used to establish hypertension and aging models. The phenotypic state was determined by detecting the marker proteins a-SM-actin, calponin, and osteopontin (OPN) via immunohistochemical staining and Western blot. Signaling proteins associated with the Akt and MAPK pathways were detected in rat thoracic aorta using Western blot. Both aging and hypertension caused a decrease in contractile (differentiated) phenotype markers (a-SM-actin and calponin), while the synthetic (proliferative or de-differentiated) phenotype maker was elevated (OPN). When combining hypertension and aging, this effect was enhanced, with Akt signaling decreased, while MAPK signaling was increased. These results suggested that VSMCs phenotype switching is modulated by a balance between Akt and MAPK signaling in the process of aging and hypertension.
\end{abstract}

\section{Key words}

Aging • Hypertension • Vascular smooth muscle cell • Phenotypic switching

\section{Corresponding author}

L. Shi, Department of Exercise Physiology, Beijing Sport University, Beijing, 100084, P. R. China. E-mail: I_j_shi72@163.com

\section{Introduction}

Aging and hypertension, which are critical risk factors contributing to cardiovascular diseases, are associated morphological and functional vascular changes characterized by endothelial dysfunction, wall thickening, reduced elasticity, and arterial stiffening. While vascular remodeling is associated with aging, its effects are enhanced by hypertension. Changes in blood pressure are closely related to blood vessel function, with vasomotor regulation mainly impacting the vascular smooth muscle cells (VSMCs). During the cardiac cycle, VSMCs are subjected to significant mechanical strain. While these cells have shown a high degree of plasticity (BochatonPiallat and Gabbiani 2005), similarly to myocardial and skeletal muscle cells, the mechanisms behind their phenotypic modulation remains unclear. Encompassed within this plasticity is an ability to alter VSMC differentiation marker profiles, a process termed phenotypic modulation or switching that is often associated with repair or several disease states (Owens et al. 2004, Sobue et al. 1999). For example, during the early stages of vasculogenesis, VSMCs are highly migratory, undergo very rapid cell proliferation, and undergo phenotypic changes in the vessel wall in response to the degree of mechanical strain, thus enabling 
them to adapt to the new conditions.

In VSMCs, phenotypic switching plays a critical role in the pathogenesis of many proliferative cardiovascular diseases, including hypertension, myocardial infarction, and coronary heart disease. During the process of phenotypic switching, protein kinase $\mathrm{B}$ (Akt) signaling has been implicated (Goncharova et al. 2002, Chol et al. 2010, Jeffery and Morrell 2002, Mandegar et al. 2004). The Akt pathway plays a critical role in many physiological and pathological processes, to include inflammatory responses, signal transduction, and cell cycle control. The most influential factor responsible for maintaining the differentiated VSMC phenotype is insulin-like growth factor-I (IGF-1), which targets the phosphoinositide 3-kinase (PI3K) and protein kinase B (Akt) pathways (Martin et al. 2007). A downstream target of Akt is the enzyme endothelial nitric oxide synthase (eNOS), which is responsible for the production of physiological amount of nitric oxide (NO) and serves as a protective agent in a large number of diseases (Li et al. 2016, Wu et al. 2011). Within the cardiovascular system, endothelial-derived NO plays a pivotal role as a key secondary messenger that regulates physiological and pathological activities (Franceschelli et al. 2017). Another important pathway in phenotypic switching is the mitogen-activated protein kinase (MAPK) pathway. MAPK signaling molecules consist of the following protein kinase families: MAPK kinase kinases (MKKKs), MAPK kinases (MKKs), and MAPKs. Of these, extracellular signal-regulated kinase (ERK) and p38 MAPK have been associated with VSMC phenotypic switching, but Jun N-terminal kinase (JNK) has not (Ma and Wells 2014, Xue et al. 2016, Harvey et al. 2016). Furthermore, Akt, ERK, and p38 MAPK signaling can target platelet-derived growth factor (PDGF), which is known to promote cell proliferation and migration (Hayashi et al. 1999). Little is known about whether these signaling alterations interact in the presence of both hypertension and aging, or if they do, if their joint modulation accelerates VSMC phenotypic switching. However, previous findings would suggest that cross-talk does occur between the Akt and MAPK pathways during phenotypic switching.

Accordingly, the primary aims of this study were to investigate if 1) hypertension superimposed with aging promotes VSMC phenotypic switching and 2) whether VSMC phenotypic modulation is associated with a balance between Akt and MAPK signaling pathway. To address these aims, spontaneously hypertensive rats
(SHR) and Wistar-Kyoto rats (WKY) were used. Blood pressure, vascular morphology, contractile proteins, and a synthetic protein were detected in the presence of aging and hypertension, to include examination of the Akt/eNOS, ERK, and p38 MAPK pathways.

\section{Materials and Methods}

\section{Animal model and sample preparation}

In this study, adult male spontaneously hypertensive rats (SHR) and Wistar-Kyoto rats (WKY) were studied at the ages of $1,3,9$, and 16 months (Okamoto 1969, Lindesay et al. 2016, Li Y. et al. 2014). All the rats were purchased from Vital River Laboratory Animal Technology Co., Ltd. (Beijing, China) and maintained at a $12: 12 \mathrm{~h}$ light-dark cycle, with a room temperature of $23-25^{\circ} \mathrm{C}$, humidity level of $40-60 \%$, and they were provided free access to standard rodent chow and water. Rats were anesthetized with pentobarbitone sodium $\left(50 \mathrm{mg} \cdot \mathrm{kg}^{-1}\right.$, intraperitoneal) and heart weight, body weight, systolic (SBP), diastolic (DBP), and heart rate (HR) were measured in warmed, restrained, conscious rats using the artery tail-cuff method (BP-2010A; Softron Biotechnology, Beijing, China). All experimental protocols pertaining to laboratory animals were approved by the Ethics Committee of Beijing Sport University and performed in accordance with Chinese animal protection laws and institutional guidelines.

\section{Histological assays}

For morphological examination, thoracic aortas were carefully dissected from the connective tissue to avoid mechanical stretching and perfused with $4 \%$ paraformaldehyde for $12 \mathrm{~h}$. The vessels were then rinsed with phosphate buffered solution, dehydrated with ethanol, embedded in paraffin, and cut into $4 \mu \mathrm{m}$ sections. These tissue sections were stained with hematoxylin and eosin (HE), and images were obtained using an inverted microscope (IX71-F22PH; Olympus, Tokyo, Japan).

\section{Immunohistochemical}

Serial thoracic aorta sections $(4 \mu \mathrm{m})$ were deparaffinized with xylene, rehydrated in graded ethanol, and pretreated with $0.01 \mathrm{M}$ citric acid buffer $(\mathrm{pH}=6)$ for $20 \mathrm{~min}$ in a thermostatic water bath $\left(99^{\circ} \mathrm{C}\right)$. All materials were pre-incubated with $3 \%$ hydrogen peroxide in phosphate-buffered saline (PBS) for $5 \mathrm{~min}$ to ensure endogenous peroxidase inactivation. The sections were 
then incubated with $0.3 \%$ TritonX-100 for $15 \mathrm{~min}$, washed 3 times in $0.01 \mathrm{M}$ PBS for $5 \mathrm{~min}$ each, and blocked with $5 \%$ normal goat serum in PBS for $20 \mathrm{~min}$. Next, the samples were incubated with anti- $\alpha$ smooth muscle actin (ab5694, Abcam, USA; 1:200), anti-Osteopontin (ab63856, Abcam, USA; 1:100), or anti-Calponin 1 (sc58707, Santa Cruz Biotechnology, USA; 1:200) primary antibodies overnight at $4{ }^{\circ} \mathrm{C}$. Following incubation, the sections were incubated with biotinylated goat anti-rabbit IgG (SA00001-2, Proteintech Group, USA) or anti-mouse IgG (SA00001-1, Proteintech Group, USA) secondary antibodies at a concentration of 1:200 in PBS for $1 \mathrm{~h}$. The sections were then washed 3 times with PBS, visualized with $0.05 \%$ 3-3'diaminobenzidine (DAB) solution, counterstained with Harris hematoxylin, and imaged on an inverted microscope (IX71-F22PH; Olympus, Tokyo, Japan). Control sections were treated with only secondary antibody.

\section{Western blot analysis}

Thoracic aortas were homogenized on ice with lysis buffer, with the supernatant collected after centrifugation. The protein concentration was determined using a Bradford assay, with bovine serum albumin (BSA) used as a standard. Equal amounts of proteins were separated on $10 \%$ SDS-polyacrylamide gels via electrophoresis and electrophoretically transferred to a polyvinylidene fluoride (PVDF) membrane. After blocking with BSA, the membrane was incubated overnight at $4{ }^{\circ} \mathrm{C}$ with the primary antibodies anti- $\alpha$ smooth muscle actin (ab5694, Abcam, USA; 1:200), anti-Osteopontin (ab63856, Abcam, USA; 1:100), antiCalponin 1 (sc58707, Santa Cruz Biotechnology, USA; 1:200), anti-Phospho-Akt (\#9271, Cell Signaling Technology, USA; 1:200), anti-Akt (\#9272, Cell Signaling Technology, USA; 1:200), anti-Phosphop44/42 MAPK (Erk1/2) (\#9101, Cell Signaling Technology, USA; 1:200), anti-p44/42 MAPK (Erk1/2) (\#9102, Cell Signaling Technology, USA; 1:200), anti-Phospho-p38 MAPK (\#9215, Cell Signaling Technology, USA; 1:200), anti-p38 MAPK (\#9212, Cell Signaling Technology, USA; 1:200), and GAPDH (sc32233, Santa Cruz Biotechnology, Santa Cruz, CA, USA; 1:500). Following the incubation, the membranes were incubated with biotinylated goat anti-rabbit $\mathrm{IgG}$ (SA00001-2, Proteintech Group, USA) or anti-mouse IgG (SA00001-1, Proteintech Group, USA) secondary antibodies at a concentration of $1: 10000$ in TBST (Tris Buffered Saline with Tween-20) for $1 \mathrm{~h}$, visualized with autoradiography using the ChemiDoc $\mathrm{XRS}^{+}$System (Bio-Rad Laboratories, Hercules, CA, USA), and the band intensities quantified using Image Lab Software (Bio-Rad Laboratories). All values are expressed as a percentage of GAPDH in each lane and then normalized to the value of 1 month.

\section{Statistical analysis}

Results are expressed as a mean \pm SEM. Normality tests were performed to determine the presence of a normal distribution, with data log-transformed in the absence of a normal distribution. Two-way analysis of variance (ANOVA) was used to assess the interactive influences of hypertension and aging. Post hoc analysis was performed using an independent sample t-test. All analyses were performed using SPSS 19.0 software, with $\mathrm{P}<0.05$ considered significant. Figures were generated using GraphPad Prism 5.

\section{Results}

Differences in blood pressure in aging and hypertensive rats

Significant differences were observed between the SHR and WKY groups, with the SHR rats having significantly increased BP (SBP and DBP) and HR when compared with the normal age-matched groups from 3 months and up. When examining the WKY groups $(n=4)$, the SBP, DBP and HR tended to be elevated with age. In particular, the SBP and mean arterial pressure showed a significant correlation with both age and hypertension $(\mathrm{P}<0.01$; Fig. 1$)$.

\section{Morphological differences}

Thoracic aorta morphological differences among groups were determined by hematoxylin and eosin staining (Fig. 2). Both hypertension and aging significantly contributed to wall thickening $(\mathrm{P}<0.01)$, with more pronounced changes seen when hypertension and aging were combined $(\mathrm{P}<0.01)$. When comparing SHR-1M and SHR-16M samples, a significant increase in thoracic aortic wall thickness was noted, with this trend was also seen in WKY samples $(\mathrm{P}<0.01)$. Moreover, this significant difference occurred as early as 9 months in the SHR groups. 
A

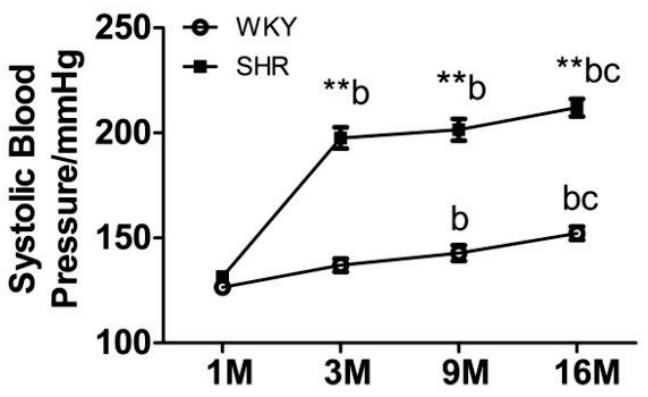

C

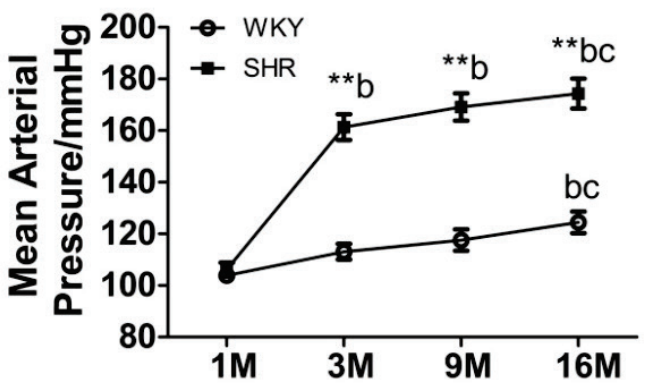

B

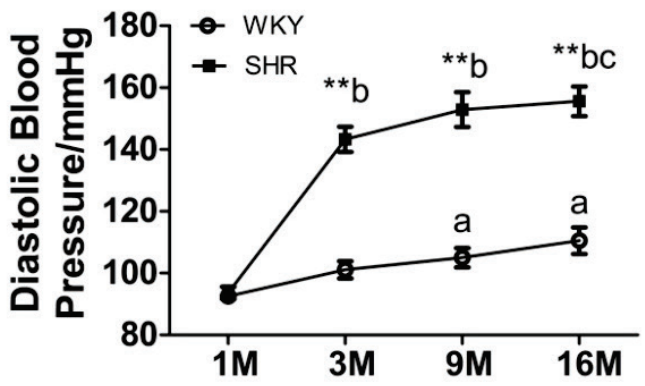

D

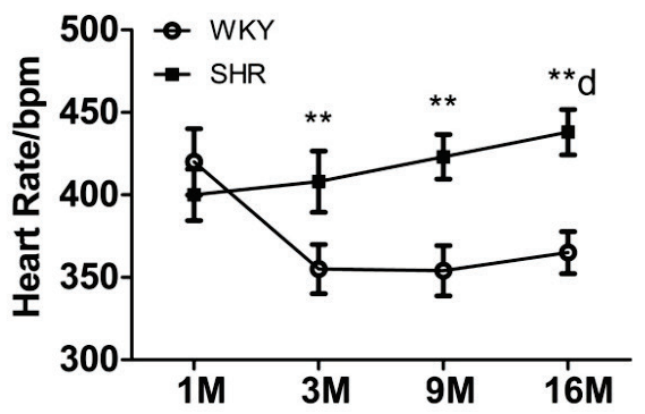

Fig. 1. Graphs showing blood pressure $(B P)$ and heart rate $(H R)$ in the eight groups. Bars indicate $S E M(n=6)$. * P<0.05 and ** $P<0.01$, sample vs. age-matched WKY; ${ }^{a} \mathrm{P}<0.05$ and ${ }^{\mathrm{b}} \mathrm{P}<0.01$, sample vs. same strain at 1 month; ${ }^{\mathrm{C}} \mathrm{P}<0.05$ and ${ }^{\mathrm{d}} \mathrm{P}<0.01$, sample vs. same strain at 3 months. (A) Systolic blood pressure (SBP); (B) diastolic blood pressure (DBP); (C) mean arterial pressure (MAP); and (D) heart rate (HR).

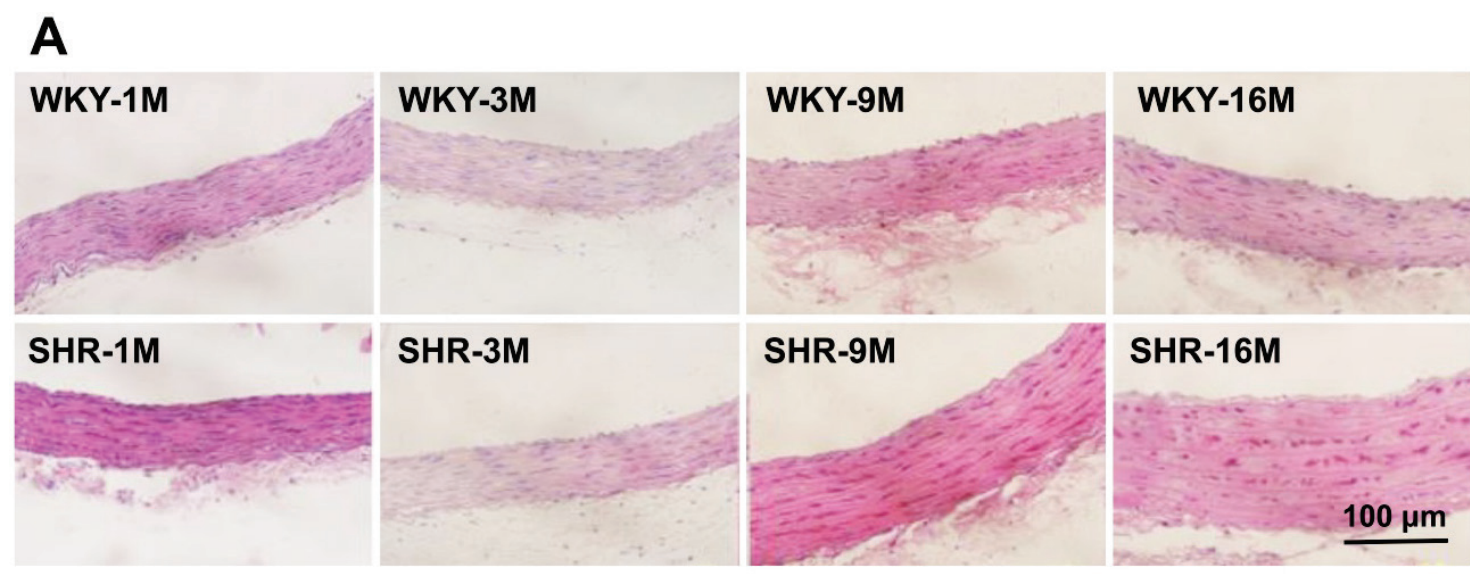

B

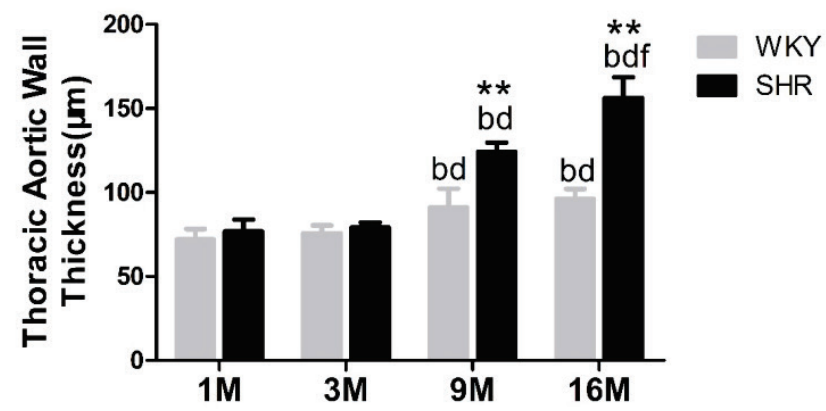

Fig. 2. Histological appearance of the thoracic aorta stained with $H \& E$ (hematoxylin and eosin). The results are expressed as a mean \pm SEM $(n=6) . * P<0.05$ and $* * P<0.01$, sample vs. age-matched $W K Y ;{ }^{a} P<0.05$ and ${ }^{b} P<0.01$, sample vs. same strain at 1 month; ${ }^{c} \mathrm{P}<0.05$ and ${ }^{\mathrm{d}} \mathrm{P}<0.01$, sample vs. same strain at 3 months; ${ }^{\mathrm{e}} \mathrm{P}<0.05$ and ${ }^{\mathrm{f}} \mathrm{P}<0.01$, sample vs. same strain at 9 months. (A) SHR and WKY thoracic aorta of different ages stained with H\&E (hematoxylin and eosin). (B) Statistical views of the thoracic aorta wall thickness in eight groups. 


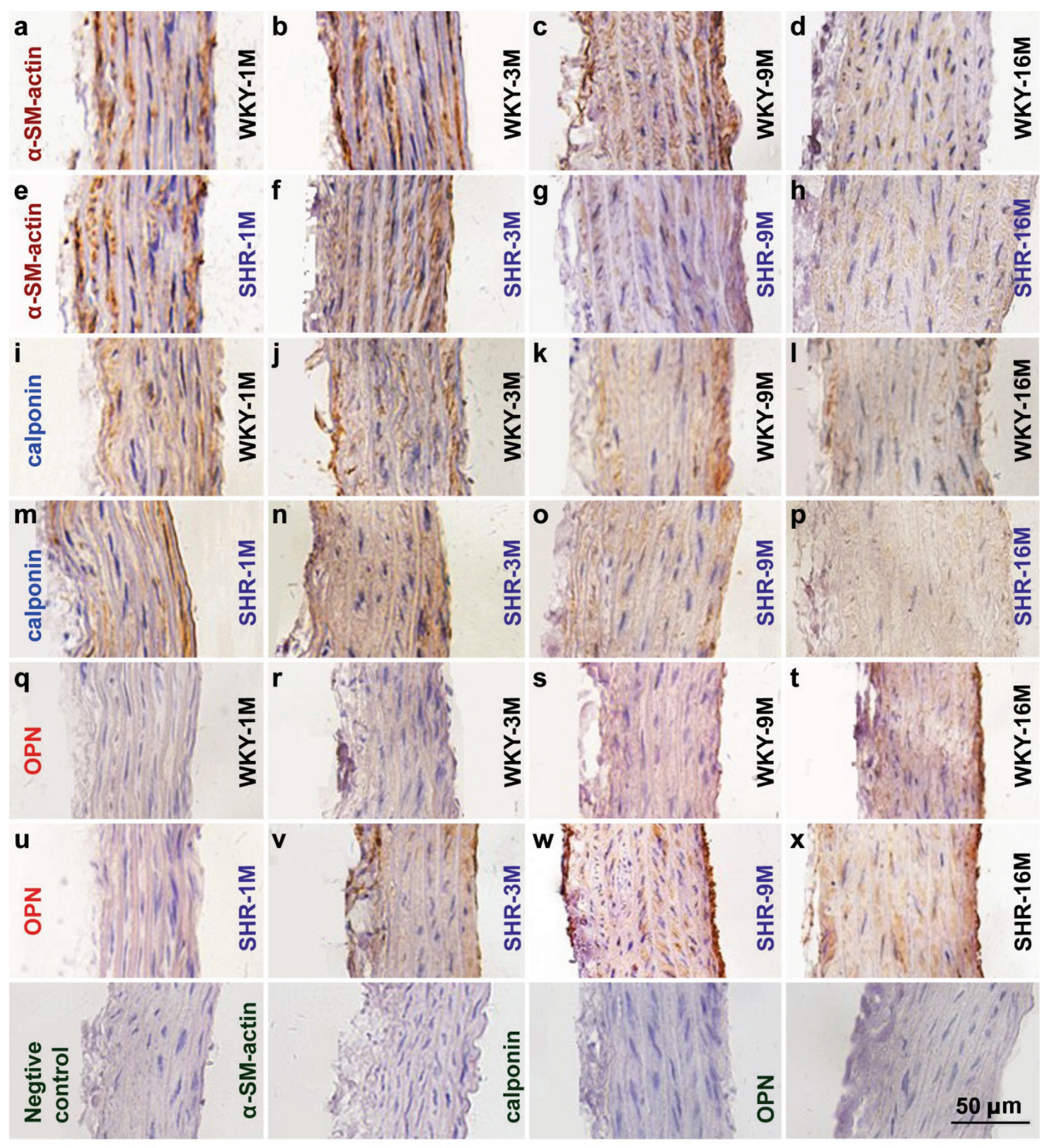

A

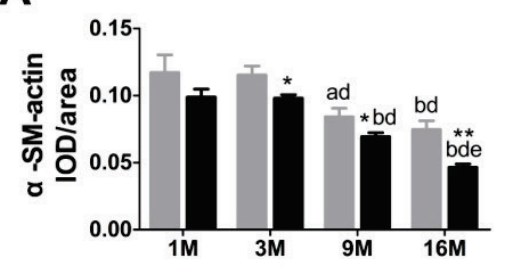

B

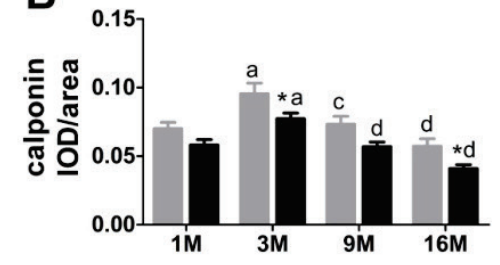

C

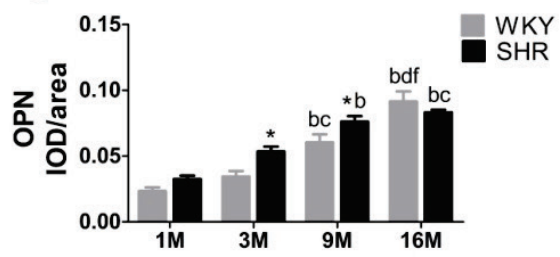

Fig. 3. Immunohistochemistry of the marker proteins in thoracic aorta smooth muscle cells. The results are expressed as a mean \pm SEM $(n=6)$. $* P<0.05$ and $* * P<0.01$, sample vs. age-matched WKY; ${ }^{a} P<0.05$ and aa $P<0.01$, sample vs. same strain at 1 month; ${ }^{\mathrm{b}} \mathrm{P}<0.05$ and ${ }^{\mathrm{bb}} \mathrm{P}<0.01$, sample vs. same strain at 3 months; ${ }^{\mathrm{c}} \mathrm{P}<0.05$ and ${ }^{c c} \mathrm{P}<0.01$, sample vs. same strain at 9 months. 
A

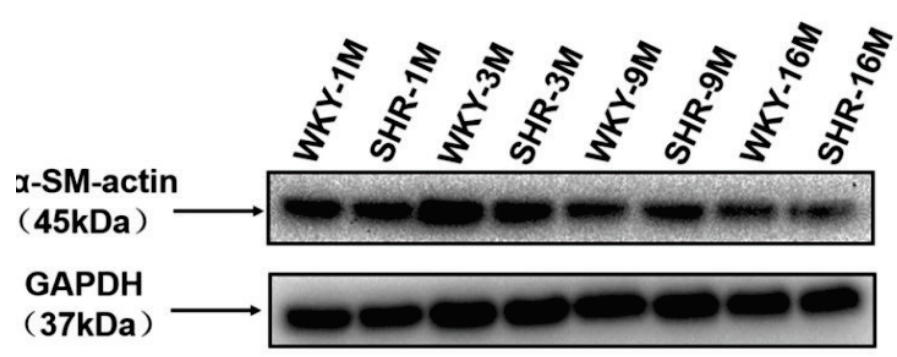

C

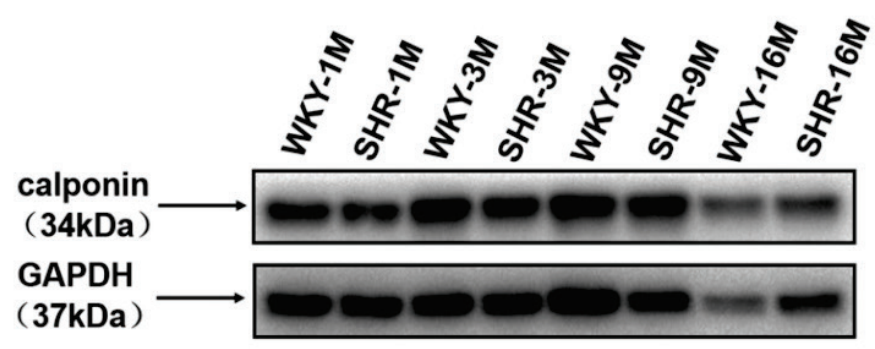

E

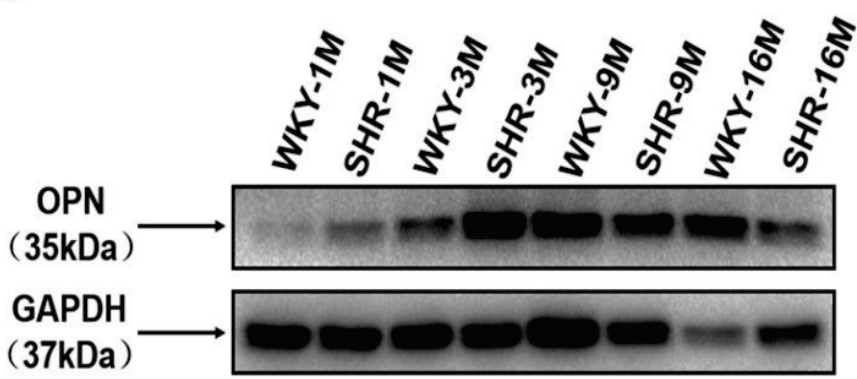

B

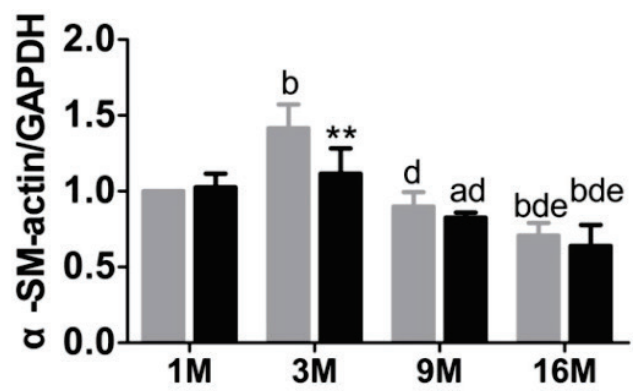

D

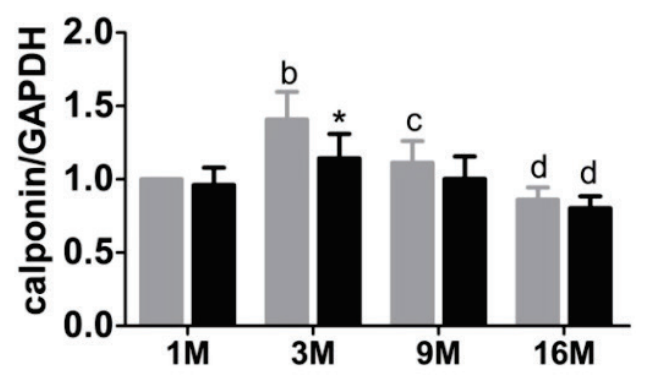

F

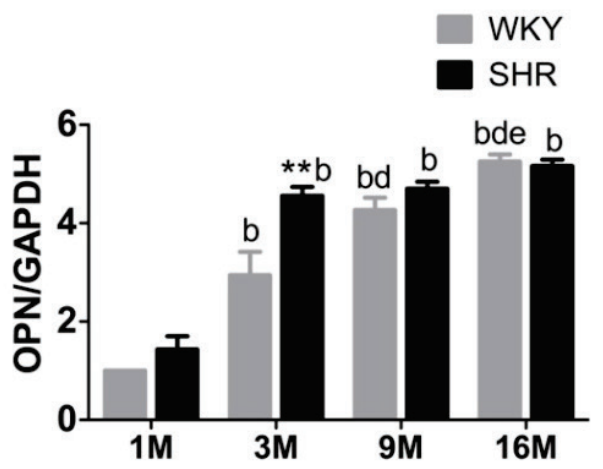

Fig. 4. Western blot of protein markers in thoracic aorta smooth muscle cells. The results are expressed as a mean $\pm S E M(n=6)$. ${ }^{*} \mathrm{P}<0.05$ and $* * \mathrm{P}<0.01$, sample vs. age-matched WKY; ${ }^{a} \mathrm{P}<0.05$ and ${ }^{\text {aa }}<0.01$, sample vs. same strain at 1 month; ${ }^{\mathrm{b}} \mathrm{P}<0.05$ and ${ }^{b b} P<0.01$, sample vs. same strain at 3 months; ${ }^{c} P<0.05$ and ${ }^{c c} P<0.01$, sample vs. same strain at 9 months. The expression levels of a-SM-actin (A), calponin (C), and OPN (E) were analyzed by Western blot. The quantitative analysis of the expression of a-SM-actin (B), calponin (D), and OPN (F) were performed by Graphpad Prism.

Examination of markers associated with phenotypic switching

Markers associated with phenotypic switching were assessed in both hypertension and aging models via immunohistochemistry and Western blot assay. When examining VSMC contractile markers both $\alpha$-SM-actin and calponin via immunohistochemistry and Western blotting, a peak were reached at the age of 3 months, followed by a progressive decrease in both samples. When comparing SHR samples with age-matched WKY samples, these contractile proteins were down-regulated in SHR thoracic aortic smooth muscle cells. When examining hypertension and aging jointly via immunohistochemisty, the expression of the contractile proteins was significant $(\alpha-S M-a c t i n: P<0.01$; calponin: $\mathrm{P}<0.01$; Figs 3a-3p, Figs 4A-4D), with $\alpha$-SM-actin also found to be significant via Western blotting $(\mathrm{P}<0.01)$.

In contrast, the synthetic marker osteopontin (OPN) was increased when examining aging and hypertension jointly (Figs 3q-3x, Figs 4E and 4F). When examining the SHR group, OPN levels were always higher than age-matched WKY samples $(3 \mathrm{M}$ : $\mathrm{P}<0.05$; $9 \mathrm{M}$ : $\mathrm{P}<0.01)$. However, when the age of 16 months was reached, no significant difference between the SHR and WKY groups was noted. 
A

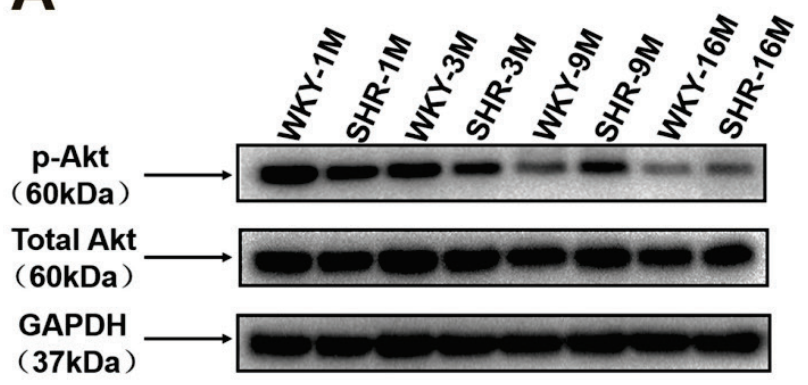

C

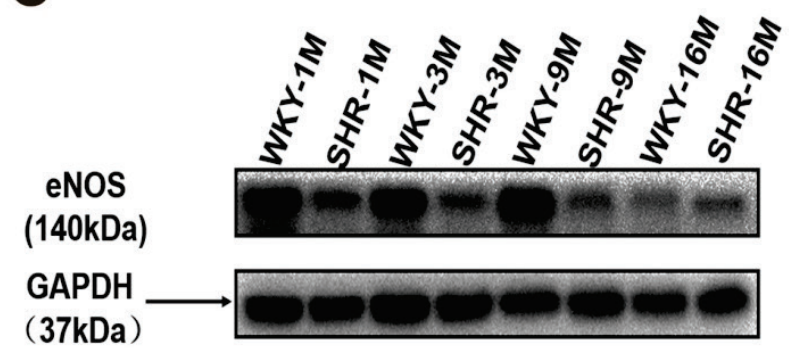

$\mathbf{E}$

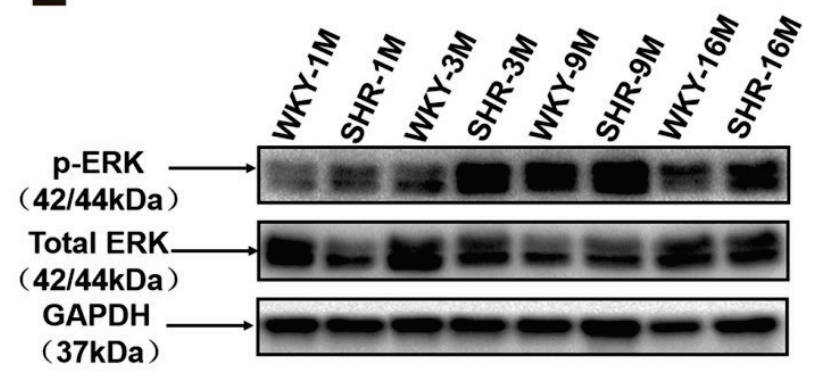

G

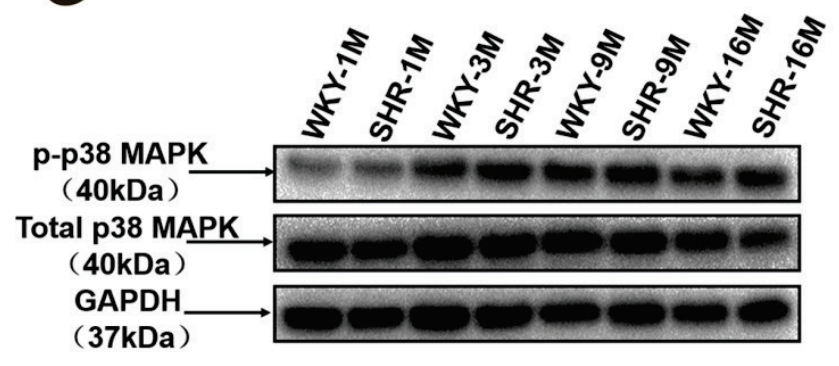

B

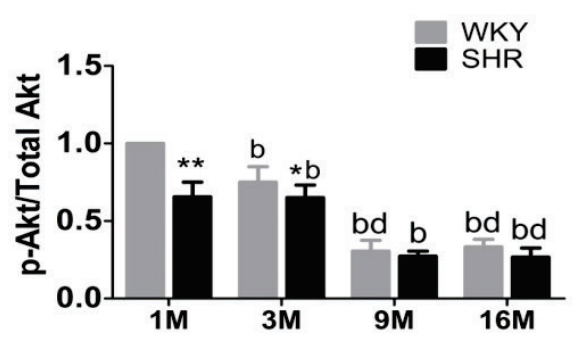

D

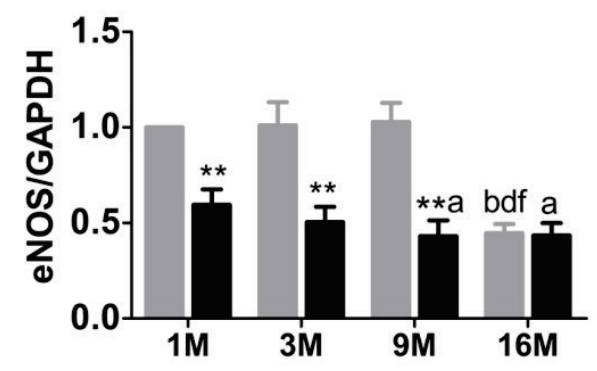

$\mathbf{F}$

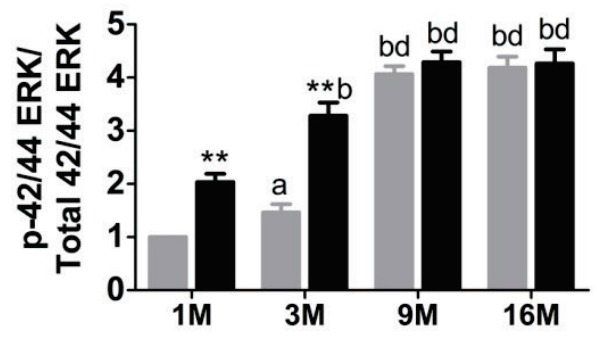

H

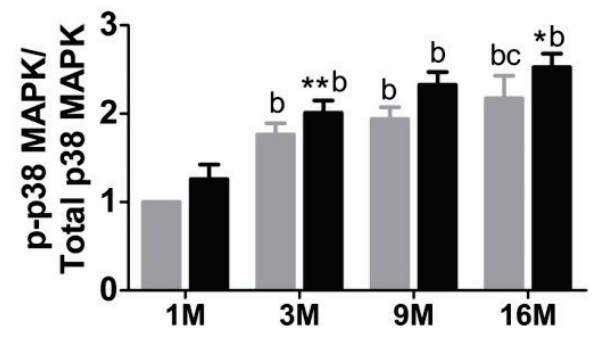

Fig. 5. Western blot of selected signaling pathways proteins in thoracic aorta smooth muscle cells. The results are expressed as a mean $\pm \operatorname{SEM}(n=6) . * P<0.05$ and $* * P<0.01$, sample vs. age-matched WKY; ${ }^{a} P<0.05$ and ${ }^{\text {aa }} P<0.01$, sample vs. same strain at 1 month; ${ }^{b} P<0.05$ and ${ }^{b b} P<0.01$, sample vs. same strain at 3 months; ${ }^{c} P<0.05$ and ${ }^{c c} P<0.01$, sample vs. same strain at 9 months. The phosphorylation of Akt $(\mathbf{A})$ and the protein expression of eNOS $(\mathbf{C})$ were reduced accompanied by the aging and hypertension. However, the phosphorylation of ERK (E) and p38 MAPK (G) were increased with aging and hypertension. The quantitative analysis of the expression of these proteins (B), (D), (F) and (H), respectively.

The expression level of signaling pathways proteins in VSMCs in hypertension and aging rats

To further analyze signal transduction relating to the thoracic aortic VSMC phenotype, AKT/eNOS signaling was examined via Western blotting. These results showed that $\mathrm{p}$-AKT and eNOS levels decreased with increasing age in both the WKY and SHR groups (Figs 5A-5D). In the SHR group, p-AKT was 
significantly down-regulated at the ages of $1(\mathrm{P}<0.01)$ and $3(\mathrm{P}<0.05)$ months compared with the age-matched WKY samples, but with no significant difference noted at the ages of 9 and 16 months. When examining eNOS expression, the SHR group showed a significant down-regulation at the ages of 1,3 , and 9 month $(\mathrm{P}<0.01)$, but no significant difference was noted at the age of 16 months.

MAPK pathway signaling was also examined, with ERK1/2 and p38 MAPK specifically probed. These proteins were found to have significantly higher expression in the SHR group relative to age-matched WKY group samples (Figs 5E-5H). When examining hypertension and aging jointly, both p-ERK $1 / 2$ and p-p38 MAPK were significantly increased. Moreover, the interactions between hypertension and aging were significant for $\mathrm{p}$-ERK1/2 $(\mathrm{P}<0.01)$, but not for p-p38 MAPK.

\section{Discussion}

In this study, hypertension and aging were examined jointly to gain insight into the mechanisms of phenotypic switching in thoracic aorta smooth muscle cells. Aging is associated with well-defined phenotypic changes that make the cardiovascular system prone to disease even in the absence of traditional risk factors (e.g. hypertension, stroke, or coronary heart disease). Similarly, hypertension strongly contributes to cardiovascular disease via phenotypic switching in VSMCs. Herein, hypertension and aging were found to accelerate phenotypic switching progression, with a more significant progression seen when these factors were combined. During hypertension and aging, the contractile protein markers expression gradually tapered. However, when examining a synthetic (proliferative) type marker, the opposite trend was seen. When examining Akt/eNOS and MAPK signaling, the Akt/eNOS pathway was decreased accompanying hypertension and aging, while ERK and p38 MAPK expression was down-regulated. These findings would suggest that the VSMC phenotype is determined by a balance between the effects of the Akt and MAPK pathways.

Hypertension and age are inseparable in the process of vascular remodeling, with both contributing to cardiovascular disease (Dan et al. 2015). Progressive changes in the structure and function of arteries occurs throughout life and includes diffuse intimal thickening and medial degeneration, increased stiffening, and reduced distensibility of large arteries (Lakatta 2015). Arterial morphology is regulated by shear stress from the pulsatile blood pressure and flow (Elvebak et al. 2010). Arterial hypertension accelerates the progression of atherosclerosis, a pathologic chronic inflammatory process of large and middle-sized arteries that shows an age-dependent progression (Barton 2010). In this study, both hypertension and aging increased rat BP, both SBP and DBP. Furthermore, thoracic aorta wall thickness was increased in both the aging and hypertension models. These results are consistent with data from both animal and human studies that indicate that superimposing aging and hypertension affects BP and vessel morphology (Kotsis et al. 2011, Sehgel et al. 2015). Herein, morphological examination revealed an enhanced medial thickness in the presence of increased age and BP.

While phenotypic switching has been implicated with VSMC dysfunction associated with hypertension, the exact mechanisms and associated signal transduction remains unclear (Kawahara et al. 2005, Laplante et al. 2003, Ushio et al. 1999). There are two phenotypic types in VSMC, contractile (differentiated) and synthetic (proliferative or de-differentiated). VSMCs change from a contractile state to a synthetic one during vascular impairment, hypertension, or atherosclerosis (Ross 1993). These two phenotypes can be distinguish using specific markers, with the contractile phenotype determined using $\alpha$-SM-actin, h1-calponin, SM22 $\alpha$, or SM-MHC, while the synthetic phenotype is determined with OPN (Baria et al. 1986, Christen et al. 1999, Han et al. 2009, Jimenez et al. 2012). In recent studies, OPN has been found to play an important role in the process of myocardial reconstruction, has been found to be up-regulated in cardiovascular disease models, and has a close association with inflammation and hypertension (Rosenberg et al. 2008, Stepien et al. 2011, Rosenberg et al. 2012, Gao et al. 2012). In the present study, SHR samples exhibited a decrease $\alpha$-SM-actin and calponin expression accompanied by increased OPN expression when compared with age-matched WKY samples. Additionally, aging WKY samples showed significantly lower $\alpha$-SM-actin and calponin expression, while OPN expression remained up-regulation (Figs 3 and 4). When superimposing aging and hypertension, the contractile markers were down-regulated, while the synthetic marker was up-regulated.

The mechanisms regulating VSMC phenotypic switching and the critical signal transduction affecting this phenotype remain controversial. While the 
PI3K/Akt/eNOS and Ras/Raf/MAPK pathways have been implicated in many cellular activities ranging from gene expression to mitosis, movement, proliferation, migration, and apoptosis (Zhu et al. 2015, Yang et al. 2013, Ouyang et al. 2014, Xue et al. 2016, Chen et al. 2015, Li H. et al. 2014), their potential roles in phenotypic switching remains unclear. In a study, the maintenance of a contractile phenotype in VSMC was shown to depend on the Akt pathway, whereas the coordinate activation of the ERK and p38 MAPK pathways induced proliferation, thus indicating that VSMC phenotype is determined by a balance between these pathways (Umemoto et al. 2006). However, how hypertension and age jointly impact the regulation of the VSMC phenotype is still unclear. Thus, this study examined these two factors jointly while monitoring the Akt/eNOS and ERK/MAPK pathways to better characterize phenotypic switching. In the SHR group, p-Akt and eNOS expression showed a downward trend when compared with age-match WKY samples. In the normal group, p-Akt and eNOS expression decreased with increasing age. Thus these findings suggest that age and hypertension when acting jointly accelerate the process of phenotypic transformation through reduced AKT signaling. When examining the ERK and p38 MAPK pathways, p-ERK and p-p38 MAPK expression was up-regulated in the SHR groups relative to the age-matched WKY group, with the same trend seen when examining just the effect of aging. Interestingly, when examining hypertension and aging jointly, a more significant change in expression was noted.
In general, this study demonstrates that hypertension and aging exert a compounded influence on the vascular system, especially relating to BP and vascular morphology. Furthermore, the Akt and MAPK pathways serve critical roles in vascular phenotypic switching during hypertension and aging. Importantly, the impacts of hypertension and aging are compounded when considered jointly and result in a reduction in Akt pathway signaling, while MAPK pathway signaling is promoted.

While previous work has focused on VSMC phenotypic modulation, this study demonstrates that the intrinsic mechanical properties of VSMCs are associated with vascular remodeling in hypertension and aging. Furthermore, the interplay between hypertension and aging may be responsible for the acceleration of vascular remodeling. In the present study, only a small number of signaling pathways pertaining to VSMC phenotype switching were examined, but other pathways relating to phenotypic modulation will be assessed in vivo and in vitro in future studies.

\section{Conflict of Interest}

There is no conflict of interest.

\section{Acknowledgements}

This work was supported by the National Natural Science Foundation of China [31771312, 31371201], the Beijing Natural Science Foundation [5172023], and the Chinese Universities Scientific Fund [2017ZD004, 2018GJ010].

\section{References}

BARIA F, COUGHLIN C, BELIN D, GABIANI G: Actin isoform synthesis and mRNA levels in quiescent and proliferating rat aortic smooth muscle cells in vivo and in vitro. Lab Invest 55: 226-233, 1986.

BARTON M: Obesity and aging: determinants of endothelial cell dysfunction and atherosclerosis. Pflugers Arch 460: 825-837, 2010.

BOCHATON-PIALLAT ML, GABBIANI G: Modulation of smooth muscle cell proliferation and migration: role of smooth muscle cell heterogeneity. Handb Exp Pharmacol 170: 645-663, 2005.

CHEN YC, WEN ZH, LEE YH, CHEN CL, HUNG HC, CHEN CH, CHEN WF, TSAI MC: Dihydroaustrasulfone alcohol inhibits PDGF-induced proliferation and migration of human aortic smooth muscle cells through inhibition of the cell cycle. Mar Drugs 13: 2390-2406, 2015.

CHOI KH, KIM JE, SONG NR, SON JE, HWANG MK, BYUN S, KIM JH, LEE KW, LEE HJ: Phosphoinositide 3-kinase is a novel target of piceatannol for inhibiting PDGF-BB-induced proliferation and migration in human aortic smooth muscle cells. Cardiovasc Res 85: 836-844, 2010.

CHRISTEN T, BOCHATON-PIALLAT ML, NEUVILLE P, RENSEN S, REDARD M, VAN EYS G, GABBIANI G: Cultured porcine coronary artery smooth muscle cells a new model with advanced differentiation. Circ Res $\mathbf{8 5}$ : 99-107, 1999. 
DAN J, SUN X, LI W, ZHANG Y, LI X, XU H, LI Z, TIAN Z, GUO S, YAO J, GAO W, TIAN Y: 5-aminolevulinic acid-mediated sonodynamic therapy promotes phenotypic switching from dedifferentiated to differentiated phenotype via reactive oxygen species and p38 mitogen-activated protein kinase in vascular smooth muscle cells. Ultrasound Med Biol 41: 1681-1689, 2015.

ELVEBAK RL, EISENACH JH, JOYNER MJ, NICHOLSON WT: The function of vascular smooth muscle phosphodiesterase III is preserved in healthy human aging. Clin Transl Sci 3: 239-242, 2010.

FRANCESCHELLI S, PESCE M, FERRONE A, GATTA DM, PATRUNO A, LUTIIS MA, QUILES JL, GRILLI A, FELACO M, SPERANZA L: Biological effect of licochalcone C on the regulation of PI3K/Akt/eNOS and $\mathrm{NF}-\kappa \mathrm{B} / \mathrm{iNOS} / \mathrm{NO}$ signaling pathways in H9c2 cells in response to LPS stimulation. Int J Mol Sci 18: pii: E690, 2017.

GAO H, STEFFEN MC, RAMOS KS: Osteopontin regulates $\alpha$-smooth muscle actin and calponin in vascular smooth muscle cells. Cell Biol Int 36: 155-161, 2012.

GONCHAROVA EA, AMMIT AJ, IRANI C, CARROLL RG, ESZTERHAS AJ, PANETTIERI RA, KRYMSKAYA VP: PI3K is required for proliferation and migration of human pulmonary vascular smooth muscle cells. Am J Physiol Lung Cell Mol Physiol 283: 354-363, 2002.

HAN M, DONG LH, ZHENG B, SHI JH, WEN JK, CHENG Y: Smooth muscle 22 alpha maintains the differentiated phenotype of vascular smooth muscle cells by inducing filamentous actin bundling. Life Sci 84: 394-401, 2009.

HARVEY A, MONTEZANO AC, LOPES RA, RIOS F, TOUYZ RM: Vascular fibrosis in aging and hypertension: molecular mechanisms and clinical implications. Can J Cardiol 32: 659-668, 2016.

HAYASHI K, TAKAHASHI M, KIMURA K, NISHIDA W, SAGA H, SOBUE K: Changes in the balance of phosphoinositide 3-kinase/protein kinase B (Akt) and the mitogen-activated protein kinases (ERK/p38MAPK) determine a phenotype of visceral and vascular smooth muscle cells. J Cell Biol 145: 727-740, 1999.

JEFFERY TK, MORRELL NW: Molecular and cellular basis of pulmonary vascular remodeling in pulmonary hypertension. Prog Cardiovasc Dis 45: 173-202, 2002.

JIMENEZ-CORONA AE, DAMIAN-ZAMACONA S, PEREZ-TORRES A, MORENO A, MAS-OLIVA J: Osteopontin upregulation in atherogenesis is associated with cellular oxidative stress triggered by the activation of scavenger receptors. Arch Med Res 43: 102-111, 2012.

KAWAHARA S, UMEMOTO S, TANAKA M, UMEJI K, MATSUDA S, KUBO M, MATSUZAKI M: Up-regulation of Akt and eNOS induces vascular smooth muscle cell differentiation in hypertension in vivo. J Cardiovasc Pharmacol 45: 367-374, 2005.

KOTSIS V, STABOULI S, KARAFILLIS I, NILSSON P: Early vascular aging and the role of central blood pressure. J Hypertens 29: 1847-1853, 2011.

LAKATTA EG: So! What's aging? Is cardiovascular aging a disease? J Mol Cell Cardiol 83: 1-13, 2015.

LAPLANTE MA, WU R, EI MIDAOUI A, DE CHAMPLAIN J: NAD(P)H oxidase activation by angiotensin II is dependent on p42/44 ERK-MAPK pathway activation in rat's vascular smooth muscle cells. J Hypertens 21: 927-936, 2003.

LI H, WANG YP, ZHANG LN, TIAN G: Perivascular adipose tissue-derived leptin promotes vascular smooth muscle cell phenotypic switching via p38 mitogen-activated protein kinase in metabolic syndrome rats. Exp Biol Med (Maywood) 239: 954-965, 2014.

LI Q, SHEN L, WANG Z, JIANG HP, LIU LX: Tanshinone IIA protects against myocardial ischemia reperfusion injury by activating the PI3K/Akt/mTOR signaling pathway. Biomed Pharmacother 84: 106-114, 2016.

LI Y, SARKAR O, BROCHU M, ANAND-SRIVASTAVA MB: Natriuretic peptide receptor-C attenuates hypertension in spontaneously hypertensive rats: role of nitroxidative stress and Gi proteins. Hypertension 63: 846-55, 2014.

LINDESAY G, RAGONNET C, CHIMENTI S, VILLENEUVE N, VAYSSETTES-COURCHAY C: Age and hypertension strongly induce aortic stiffening in rats at basal and matched blood pressure levels. Physiol Rep 4: pii: e12805, 2016.

MA B, WELLS A: The mitogen-activated protein (MAP) kinases p38 and extracellular signal-regulated kinase (ERK) are involved in hepatocyte-mediated phenotypic switching in prostate cancer cells. J Biol Chem 289: 11153-11161, 2014. 
MANDEGAR M, FUNG YC, HUANG W, REMILLARD CV, RUBIN LJ, YUAN JX: Cellular and molecular mechanisms of pulmonary vascular remodeling: role in the development of pulmonary hypertension. Microvasc Res 68: 75-103, 2004.

MARTIN KA, MERENICK BL, DING M, FETALVERO KM, RZUCIDLO EM, KOZUL CD, BROWN DJ, CHIU HY, SHYU M, DRAPEAU BL, WAGNER RJ, ROWELL RJ: Rapamycin promotes vascular smooth muscle cell differentiation through insulin receptor substrate-1/phosphatidylinositol 3-kinase/Akt2 feedback signaling. J Biol Chem 282: 36112-36120, 2007.

OKAMOTO K: Spontaneous hypertension in rats. Int Rev Exp Pathol 7: 227-270, 1969.

OUYANG QF, HAN Y, LIN ZH, XIE H, XU CS, XIE LD: Fluvastatin upregulates the $\alpha 1 \mathrm{C}$ subunit of CaV1.2 channel expression in vascular smooth muscle cells via RhoA and ERK/p38 MAPK pathways. Dis Markers 2014: $237067,2014$.

OWENS GK, KUMAR MS, WAMHOFF BR: Molecular regulation of vascular smooth muscle cell differentiation in development and disease. Physiol Rev 84: 767-801, 2004.

ROSENBERG M, ZUQCK C, NELLES M, JUENQER C, FRANK D, REMPPIS A, GIANNITSIS E, KATUS HA, FREY N: Osteopontin, a new prognostic biomarker in patients with chronic heart failure. Circ Heart Fail 1: 43-49, 2008.

ROSENBERG M, MEYER FJ, GRUENIG E, SCHUSTER T, LUTZ M, LOSSNITZER D, WIPPLINGER R, KATUS HA, FREY N: Osteopontin (OPN) improves risk stratification in pulmonary hypertension (PH). Int J Cardiol 155: 504-505, 2012.

ROSS R: The pathogenesis of atherosclerosis: a perspective for the 1990s. Nature 362: 801-809, 1993.

SEHGEL NL, SUN Z, HONG Z, HUNTER WC, HILL MA, VATNER DE, VATNER SF, MEININGER GA: Augmented vascular smooth muscle cell stiffness and adhesion when hypertension is superimposed on aging. Hypertension 65: 370-377, 2015.

SOBUE K, HAYASHI K, NISHIDA W: Expressional regulation of smooth muscle cell-specific genes in association with phenotypic modulation. Mol Cell Biochem 190: 105-118, 1999.

STEPIEN E, WYPASEK E, STOPYRA K, KONIECZYNSKA M, PRZYBYLO M, PASOWICZ M: Increased levels of bone remodeling biomarkers (osteoprotegerin and osteopontin) in hypertensive individuals. Clin Biochem 44: 826-831, 2011.

UMEMOTO S, KAWAHARA S, HASHIMOTO R, UMEJI K, MATSUDA S, TANAKA M, KUBO M, MATSUZAKI $\mathrm{M}$ : Different effects of amlodipine and enalapril on the mitogen-activated protein kinase/extracellular signalregulated kinase kinase-extracellular signal-regulated kinase pathway for induction of vascular smooth muscle cell differentiation in vivo. Hypertens Res 29: 179-186, 2006.

USHIO-FUKAI M, ALEXANDER RW, AKERS M, YIN Q, FUJIO Y, WALSH K, GRIENDLING KK: Reactive oxygen species mediate the activation of Akt/Protein kinase B by angiotensin II in vascular smooth muscle cells. J Biol Chem 274: 22699-22704, 1999.

WU Y, XIA ZY, MENG QT, ZHU J, LEI S, XU J, DOU J: Shen-Fu injection preconditioning inhibits myocardial ischemia-reperfusion injury in diabetic rats: activation of eNOS via the PI3K/Akt pathway. $J$ Biomed Biotechnol 2011: 384627, 2011.

XUE XH, ZHOU XM, WEI W, CHEN T, SU QP, TAO J, CHEN LD: Alisol a 24-acetate, a triterpenoid derived from Alisma orientale, inhibits Ox-LDL-induced phenotypic transformation and migration of rat vascular smooth muscle cells through suppressing ERK1/2 signaling. J Vasc Res 53: 291-300, 2016.

YANG X, GONG Y, TANG Y, LI H, HE Q, GOWER L, LIAW L, FRIESEL RE: Spry1 and Spry4 differentially regulate human aortic smooth muscle cell phenotype via Akt/FoxO/myocardin signaling. PloS One 8: e58746, 2013.

ZHU SB, ZHU J, ZHOU ZZ, XI EP, WANG RP, ZHANG Y: TGF- $\beta 1$ induces human aortic vascular smooth muscle cell phenotype switch through PI3K/AKT/ID2 signaling. Am J Transl Res 7: 2764-2774, 2015. 